\title{
Synthesis of somatostatin mimetics based on the 1- deoxymannojirimycin scaffold
}

Sebastien G. Gouin and Paul V. Murphy*

Centre for Synthesis and Chemical Biology,

UCD School of Chemistry and Chemical Biology, Conway Institute, University College Dublin, Belfield, Dublin 4, Ireland.

paul.v.murphy@ucd.ie

\section{Supporting Information}

1. General Experimental Conditions...................................... 2

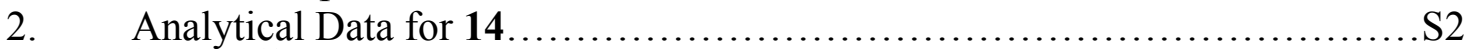

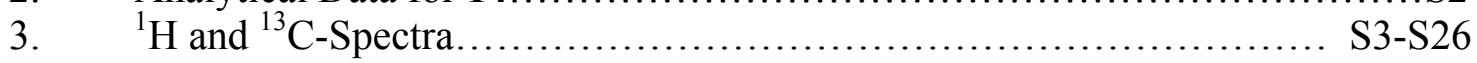




\section{General Experimental Conditions}

Chemical shifts are reported relative to internal $\mathrm{Me}_{4} \mathrm{Si}$ in $\mathrm{CDCl}_{3}(\delta 0.0)$ and $(\delta 77.16)$ for ${ }^{13} \mathrm{C}$ or HOD for $\mathrm{D}_{2} \mathrm{O}(\delta 4.79)$ for ${ }^{1} \mathrm{H}$ or $\left(\mathrm{CHD}_{2} \mathrm{CD}_{3}\right)_{2} \mathrm{SO}$ for DMSO-d $\mathrm{d}_{6}(\delta 2.50)$ for ${ }^{1} \mathrm{H}$ and $(\delta 39.52)$ for

${ }^{13} \mathrm{C} .{ }^{13} \mathrm{C}$ signals were assigned with the aid of DEPT- $135 .{ }^{1} \mathrm{H}$ signals were assigned with the aid of COSY. TLC was performed on aluminium sheets precoated with Silica Gel 60 (HF254, E. Merck) and spots visualized by UV and charring with 1:20 $\mathrm{H}_{2} \mathrm{SO}_{4}$-EtOH. Flash column chromatography was carried out with Silica Gel $60(0.040-0.630 \mathrm{~mm}$, E. Merck) and employed a stepwise solvent polarity gradient correlated with the TLC mobility. Chromatography solvents used were $\mathrm{MeOH}, \mathrm{CH}_{2} \mathrm{Cl}_{2}$ (Riedel-de Haen), EtOAc and cyclohexane (Fluka). Reaction solvents were dried and distilled before use.

Analytical data for $14: \quad[\alpha]_{\mathrm{D}}+22\left(c 0.5, \mathrm{CDCl}_{3}\right) ;{ }^{1} \mathrm{H}-\mathrm{NMR}\left(\mathrm{CDCl}_{3}, 300 \mathrm{MHz}\right): \delta(\mathrm{ppm}) 4.96$ (1H, d, J 7Hz, CHH’OMe), 4.66 (1H, d, J 7Hz, CHH’OMe), 4.45 (1H, dd, J $\mathrm{J}_{3,4} 9 \mathrm{~Hz}, \mathrm{~J}_{4,5} 8 \mathrm{~Hz}, \mathrm{H}-$ 4), 4.28-4.17 (3H, m, H-1,2,3), $4.13\left(1 \mathrm{H}, \mathrm{dd}, \mathrm{J}_{5,6} 5 \mathrm{~Hz}, \mathrm{~J}_{6,6}, 7 \mathrm{~Hz}, \mathrm{H}-6\right), 3.65\left(1 \mathrm{H}, \mathrm{dd}, \mathrm{J}_{5,6}, 9 \mathrm{~Hz}, \mathrm{~J}_{6,6}\right.$ ' 7Hz, H-6'), 3.17 (1H, ddd, $\mathrm{J}_{4,5} 8 \mathrm{~Hz}, \mathrm{~J}_{5,6} 5 \mathrm{~Hz}, \mathrm{~J}_{5,6}$ ' $\left.9 \mathrm{~Hz}, \mathrm{H}-5\right), 3.39$ (3H, m, $\left.\mathrm{CH}_{2} \mathrm{OCH}_{3}\right), 3.25(1 \mathrm{H}$, dd, $\left.\mathrm{J}_{1,1}, 15 \mathrm{~Hz}, \mathrm{~J}_{1,2} 3 \mathrm{~Hz}, \mathrm{H}-1^{\prime}\right), 1.51\left(3 \mathrm{H}, \mathrm{s}, \mathrm{CH}_{3}\right), 1.36\left(3 \mathrm{H}, \mathrm{s}, \mathrm{CH}_{3}\right) ;{ }^{13} \mathrm{C}-\mathrm{NMR}\left(\mathrm{CDCl}_{3}, 75 \mathrm{MHz}\right)$ : $\delta(\mathrm{ppm}) 157.2(\mathrm{~s}, \mathrm{C}=\mathrm{O}), 109.7(\mathrm{~s}), 96.6(\mathrm{t}), 78.3,76.4,72.7($ each d), $66.0(\mathrm{t}), 55.9(\mathrm{q}), 55.1,40.8$ (each d), 27.8, 26.0 (each q); ES-HRMS: Found $274.1291[\mathrm{M}+\mathrm{H}]^{+} ; \mathrm{C}_{12} \mathrm{H}_{20} \mathrm{NO}_{6}$ requires 274.1291. 


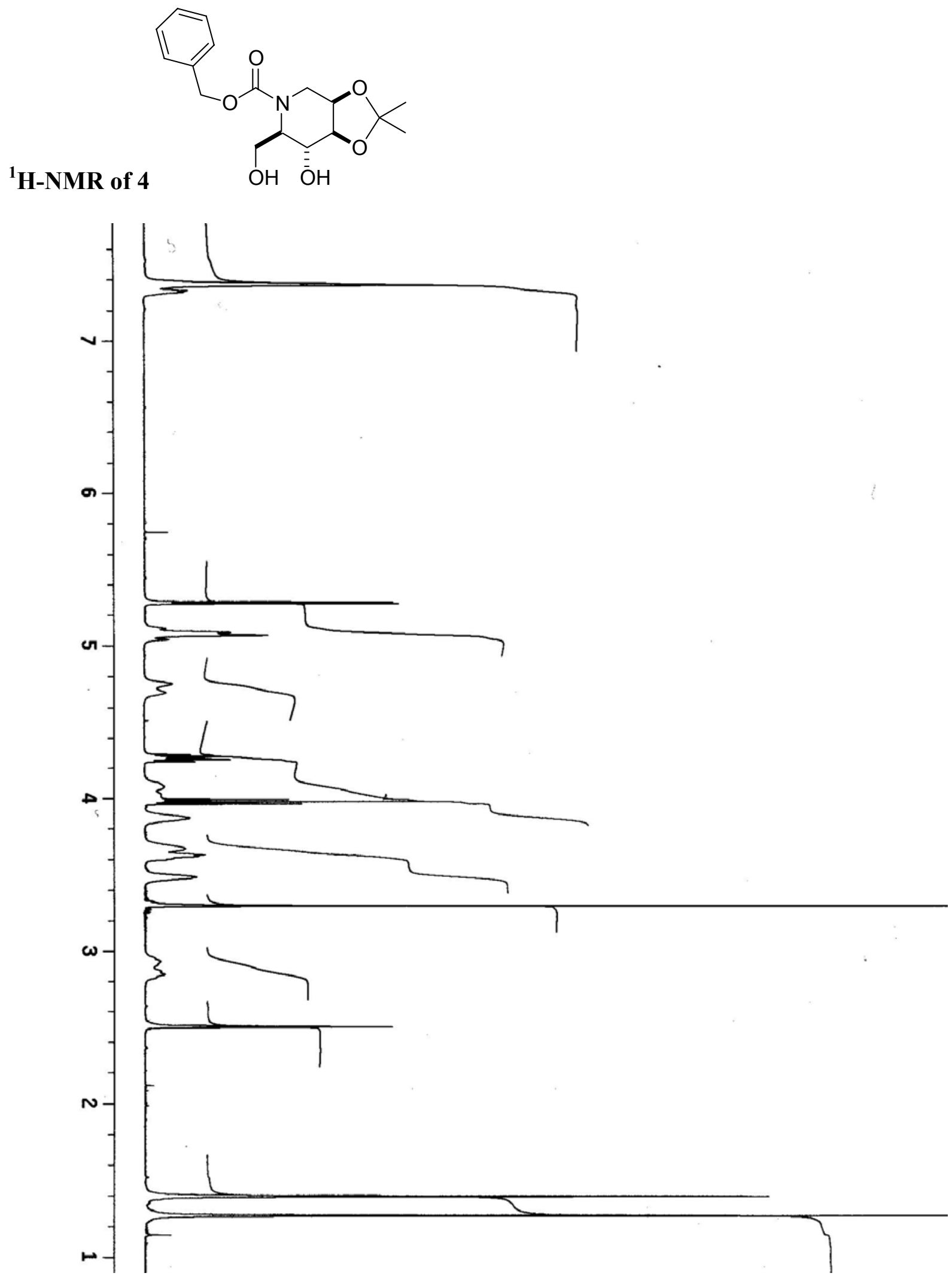




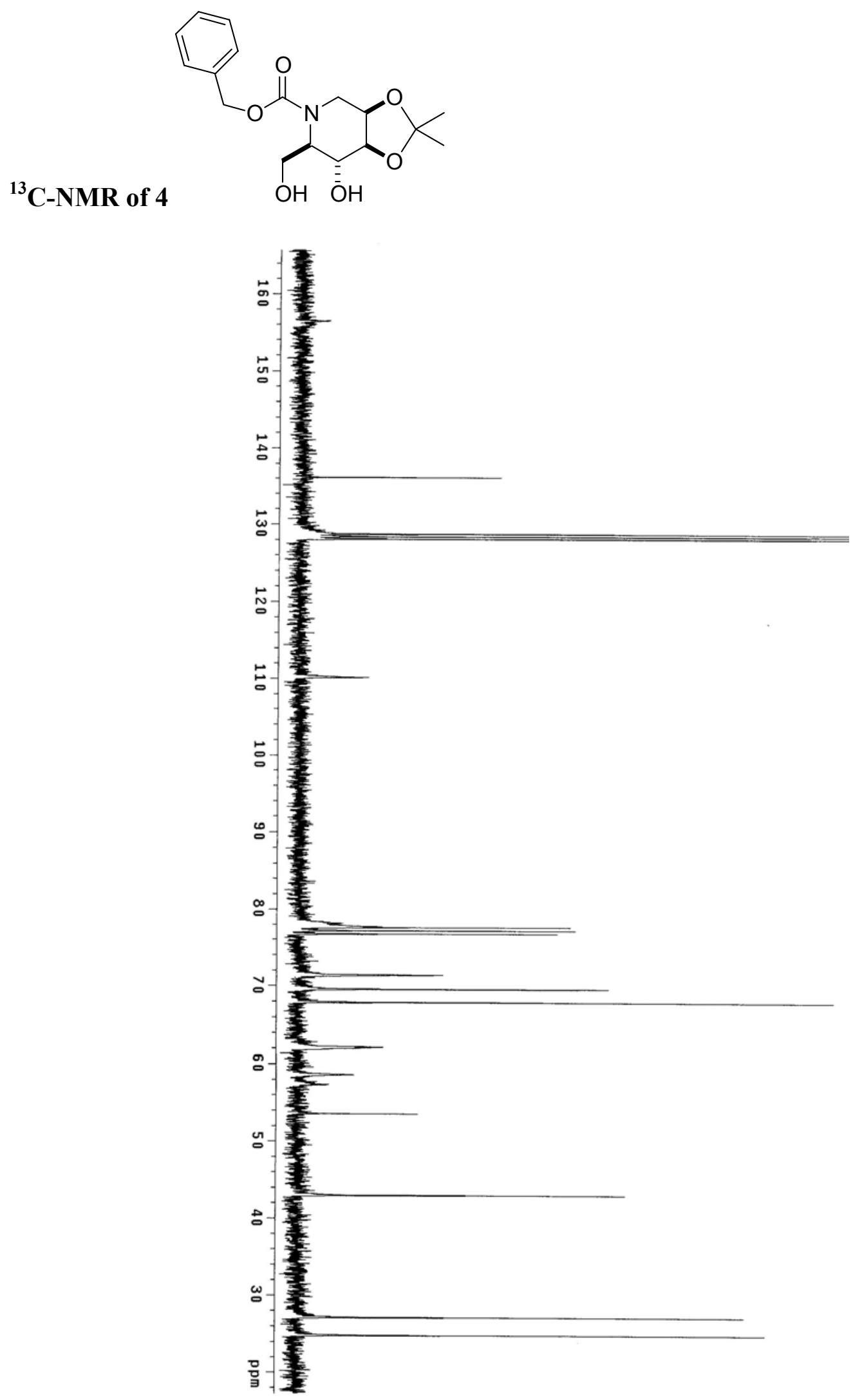



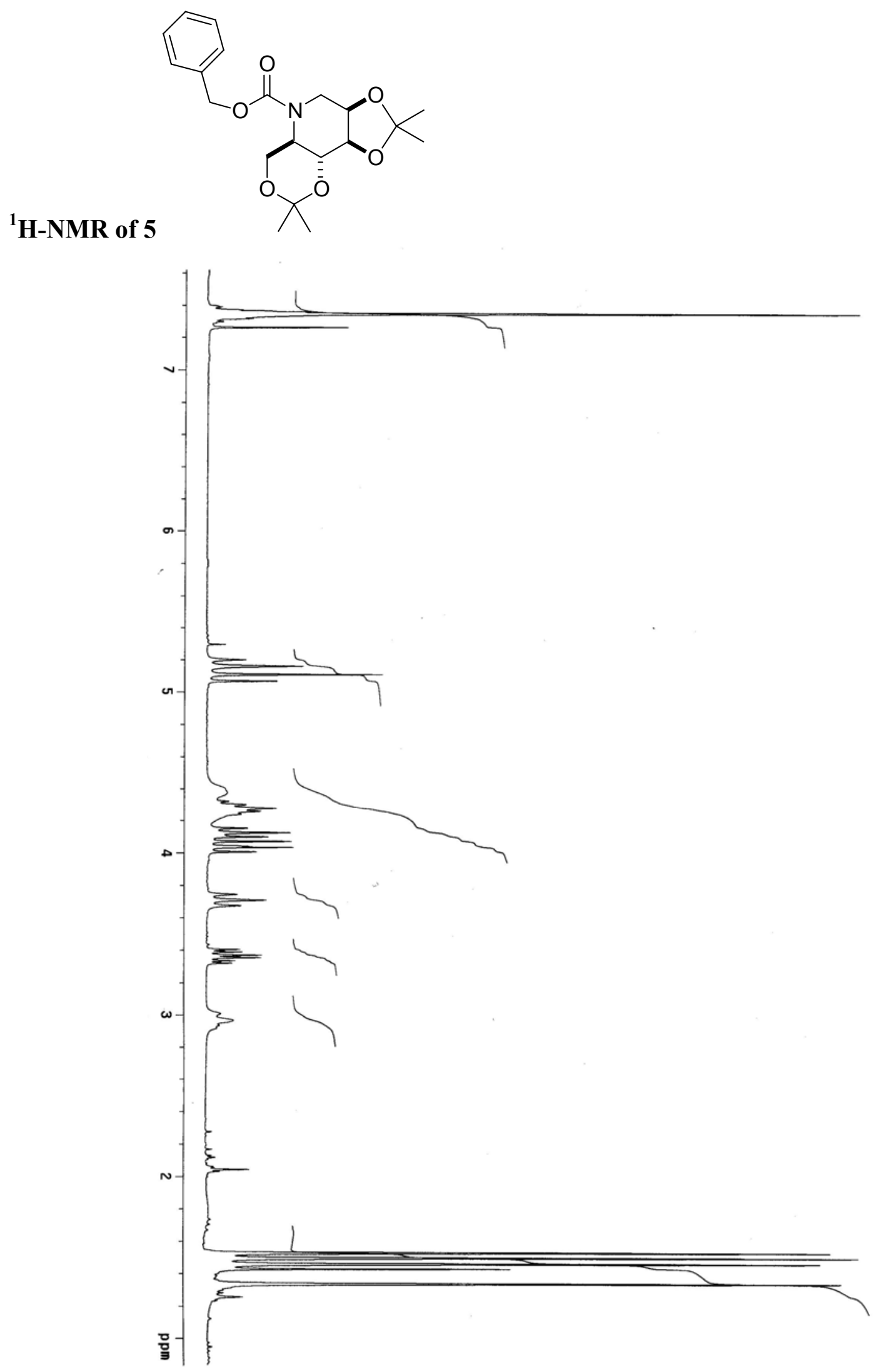

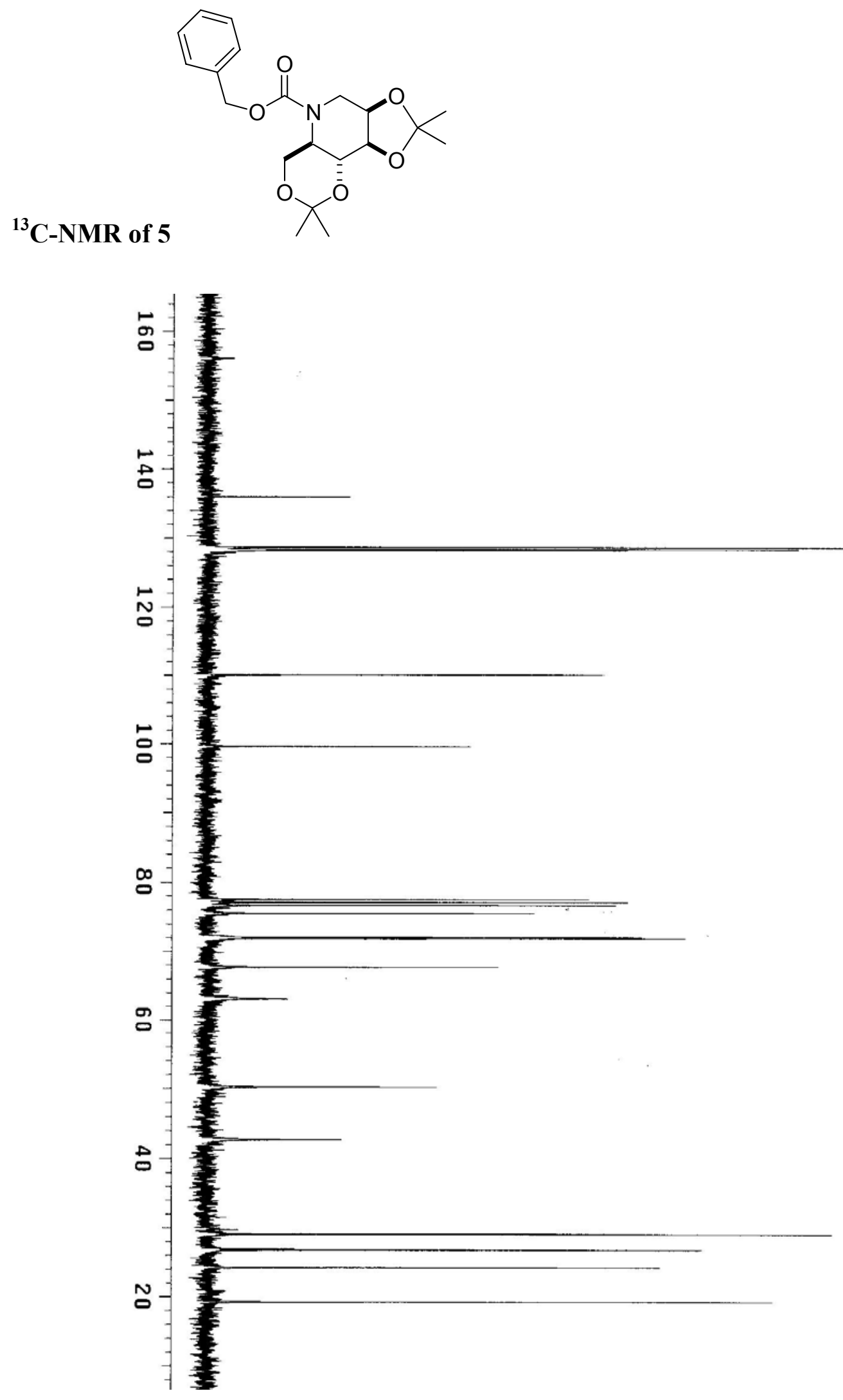

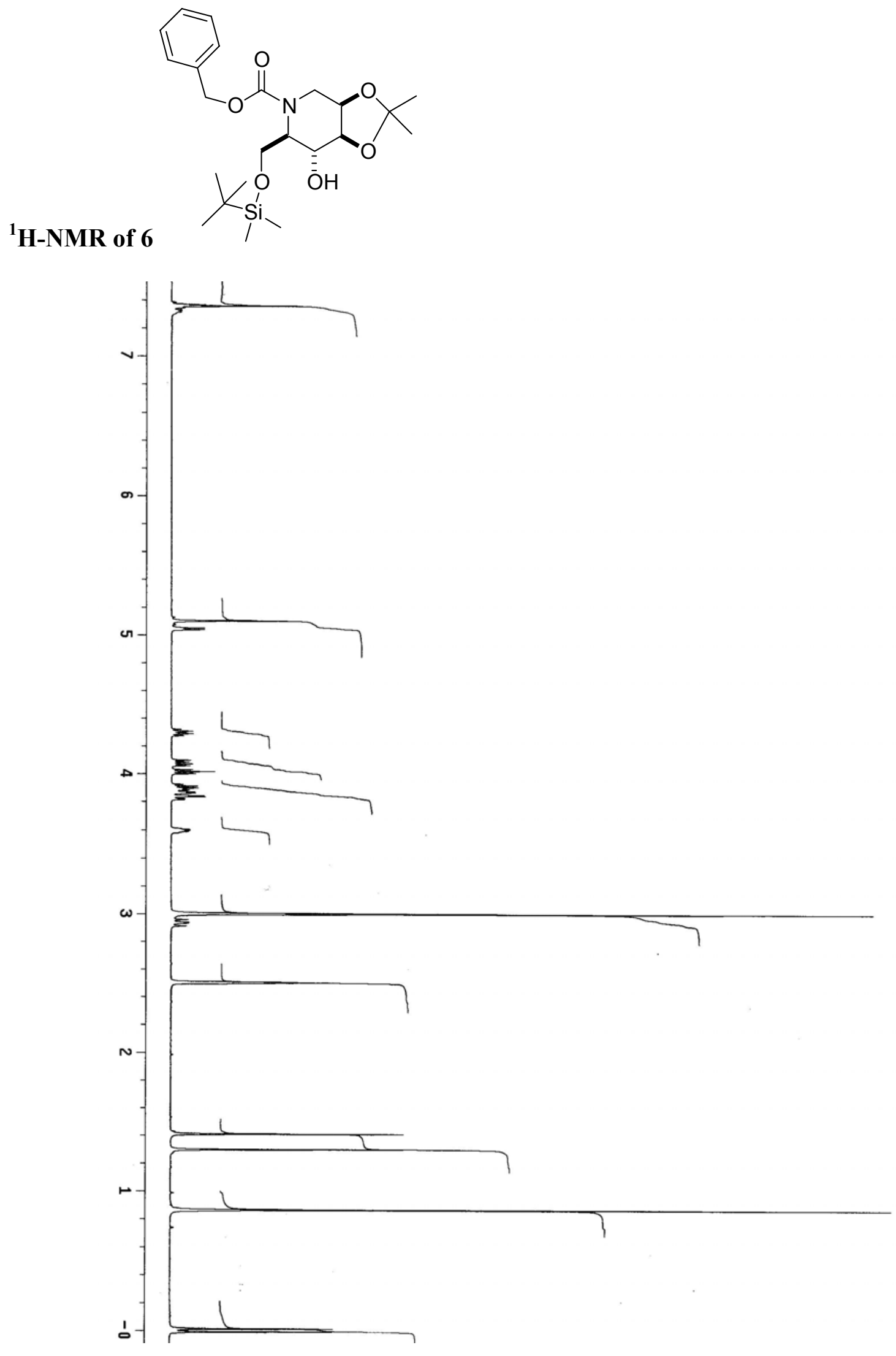
${ }^{13} \mathrm{C}$-NMR of $6\left(90{ }^{\circ} \mathrm{C}\right.$, DMSO- $\left.d_{6}\right)$<smiles>CC1(C)OC2CN(C(=O)OCc3ccccc3)[C@H](CO[Si](C)(C)C(C)(C)C)[C@H](O)C2O1</smiles>

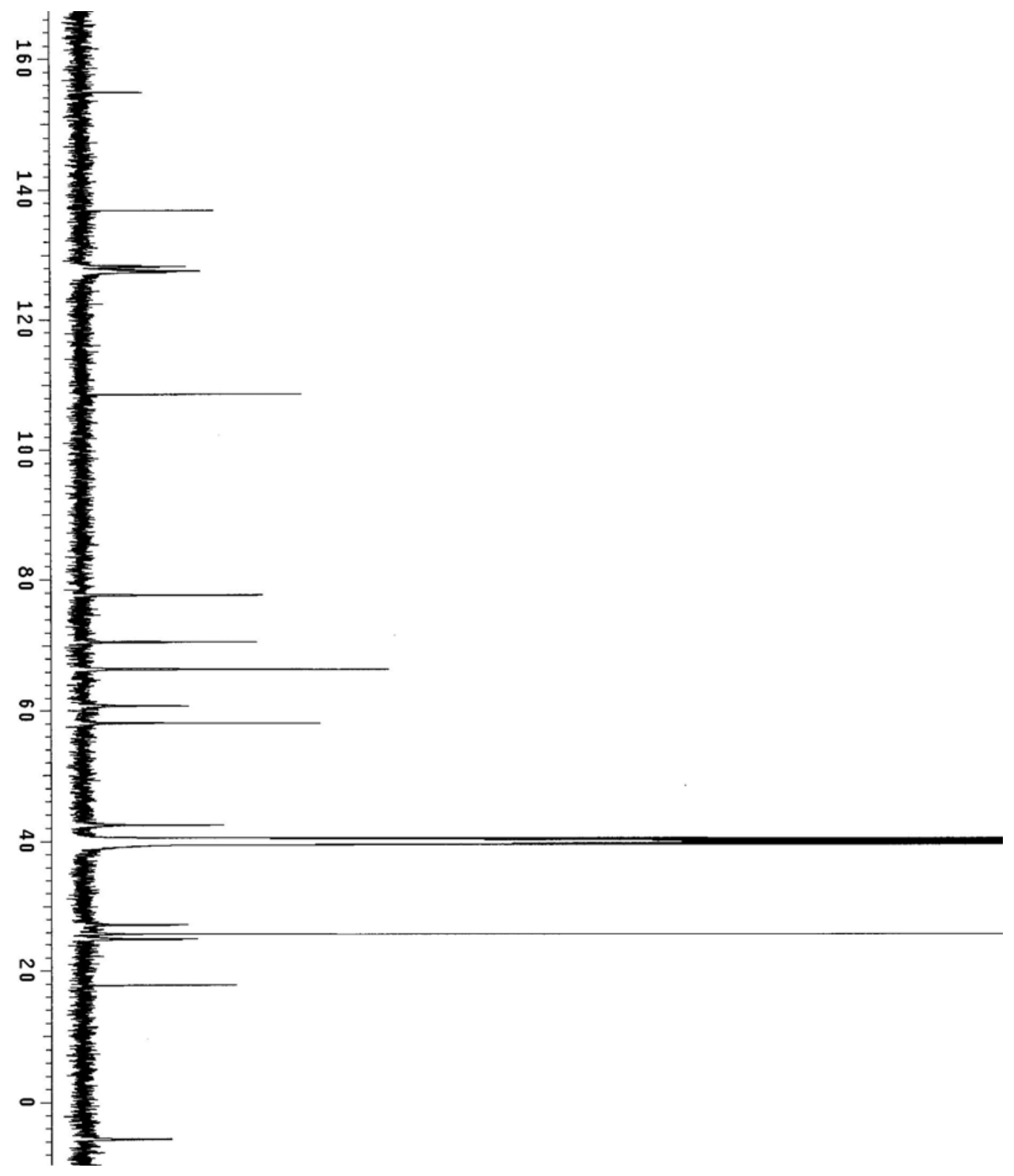




\section{${ }^{1}$ H-NMR of 7}
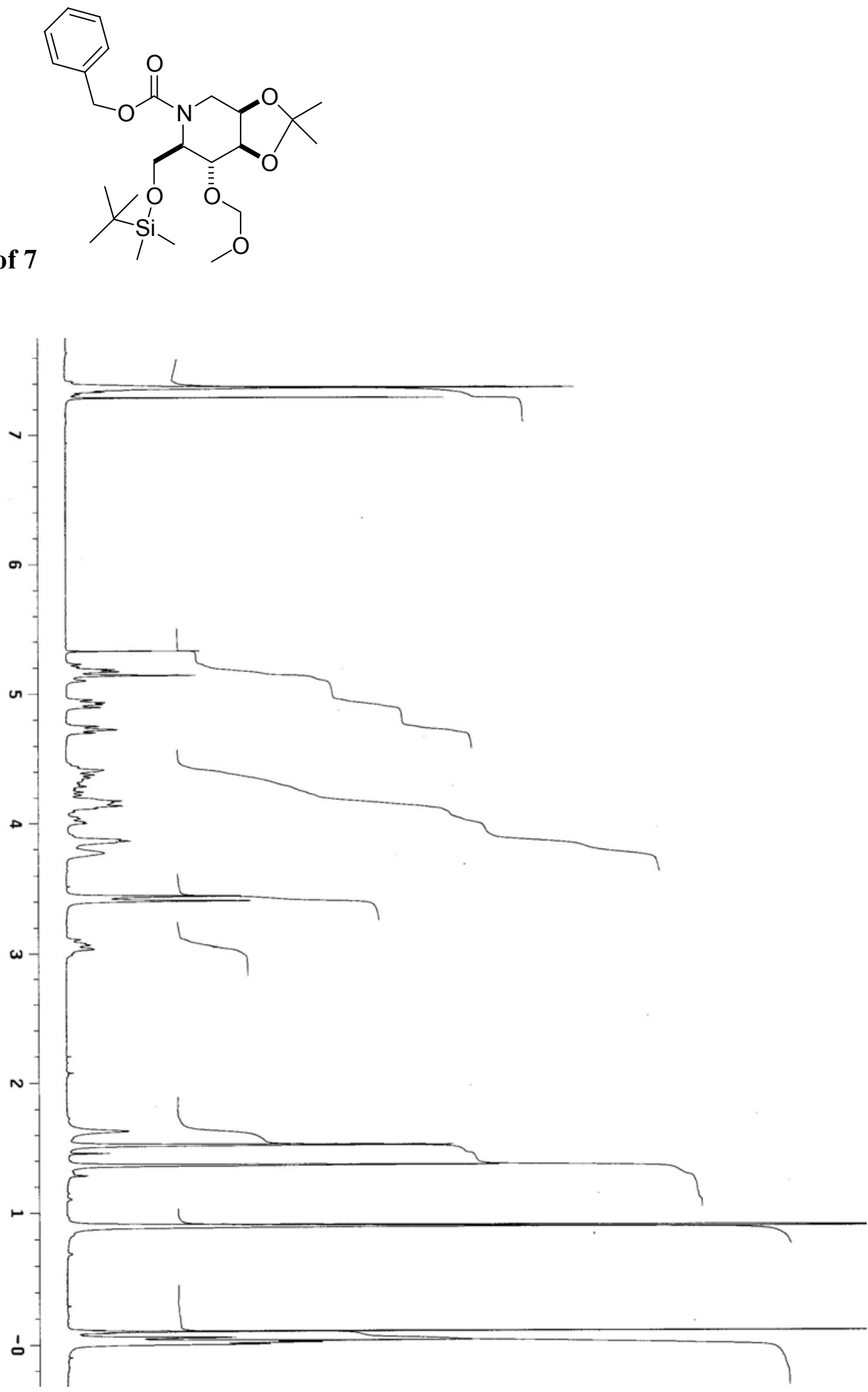

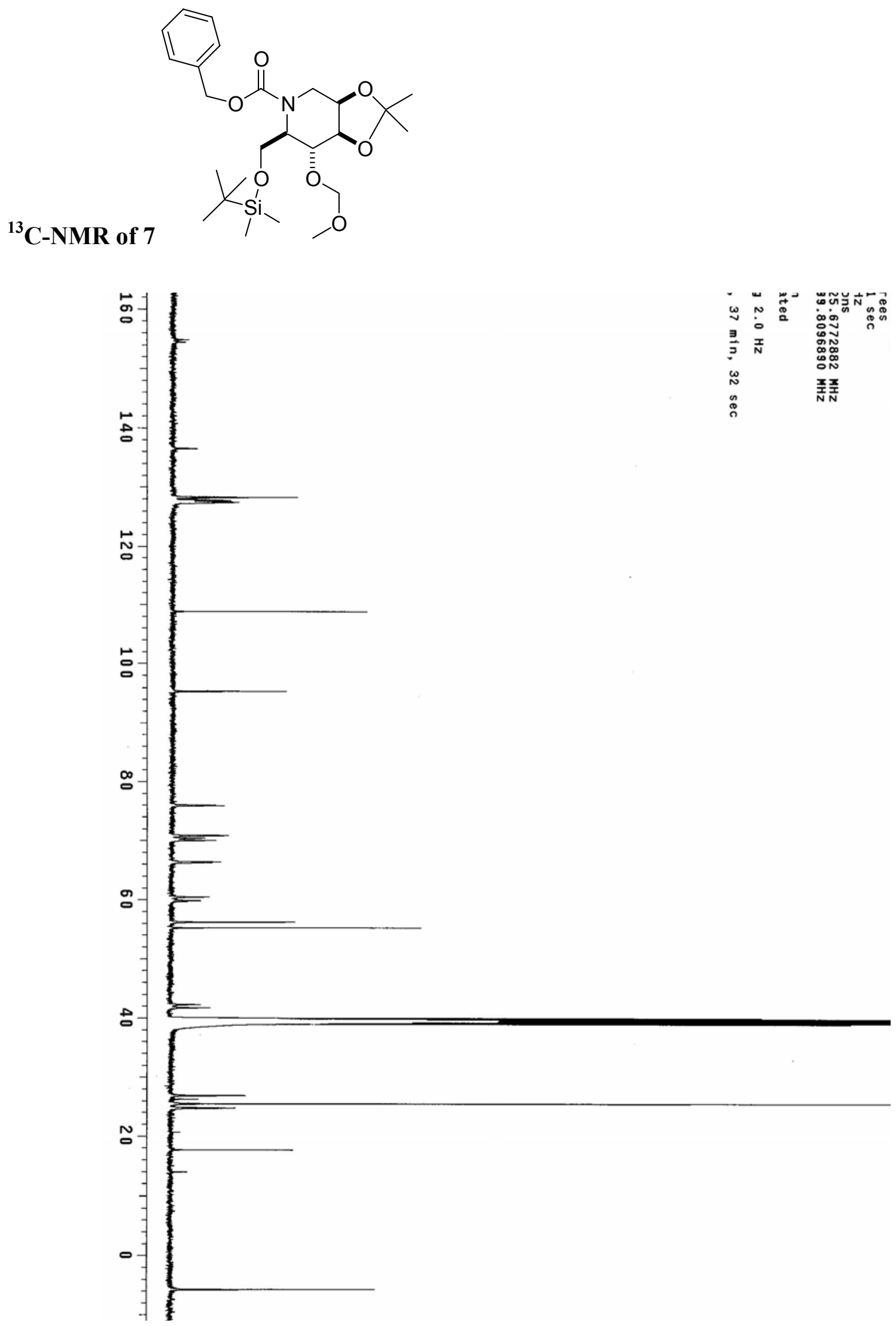


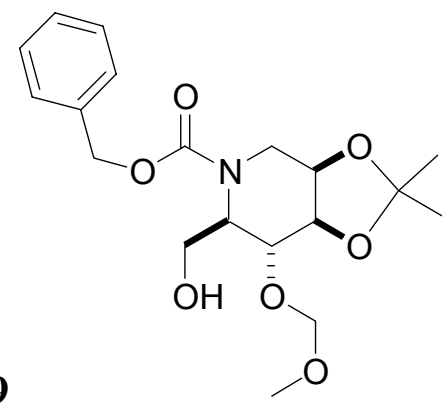

${ }^{1}$ H-NMR of 9

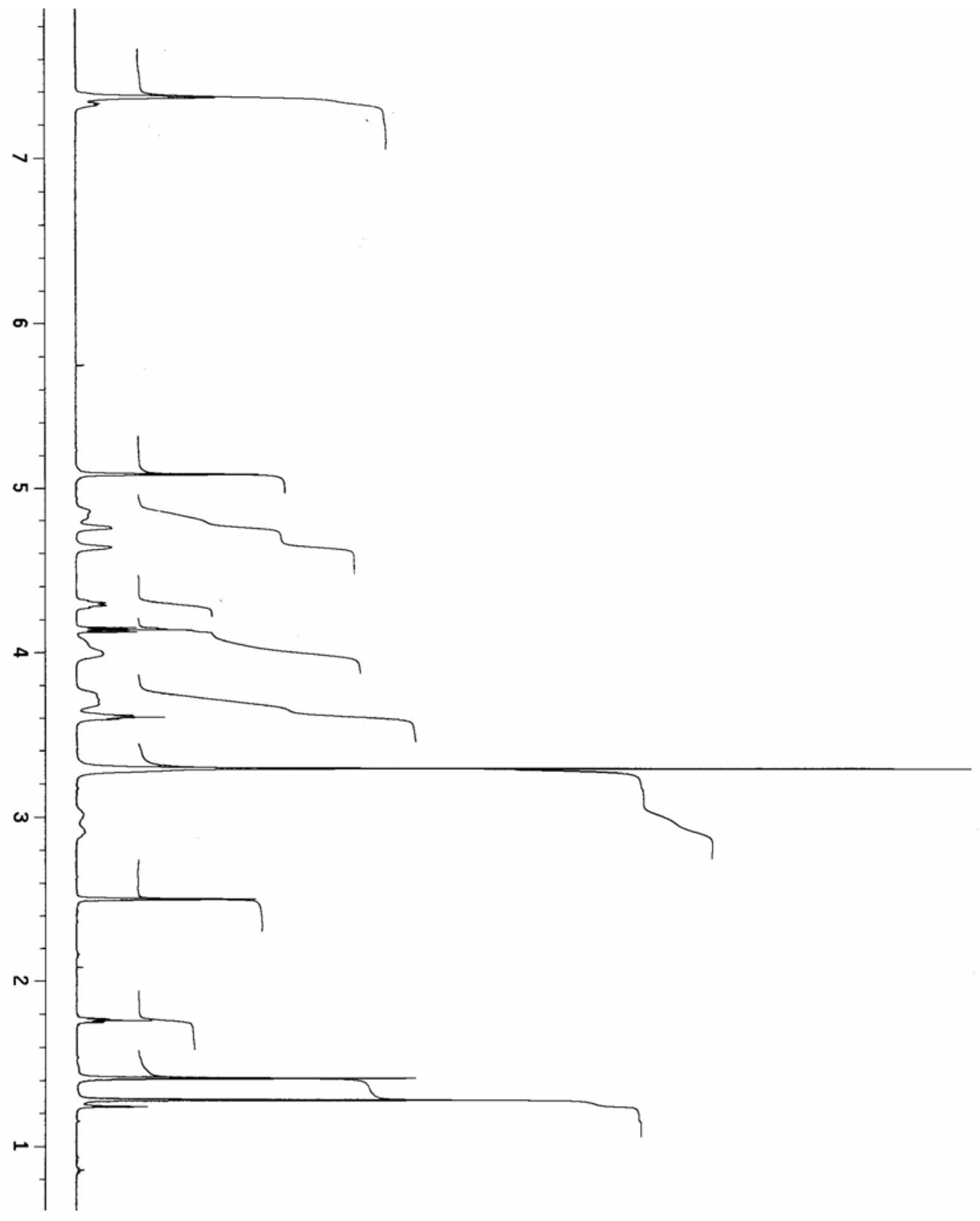


Somatostatin mimetics based 1-deoxymannojirimycin

Gouin and Murphy

$\mathrm{S} 12$

${ }^{13}$ C-NMR of 9<smiles>COCO[C@H]1C2OC(C)(C)O[C@@H]2CN(C(=O)OCc2ccccc2)[C@@H]1CO</smiles>

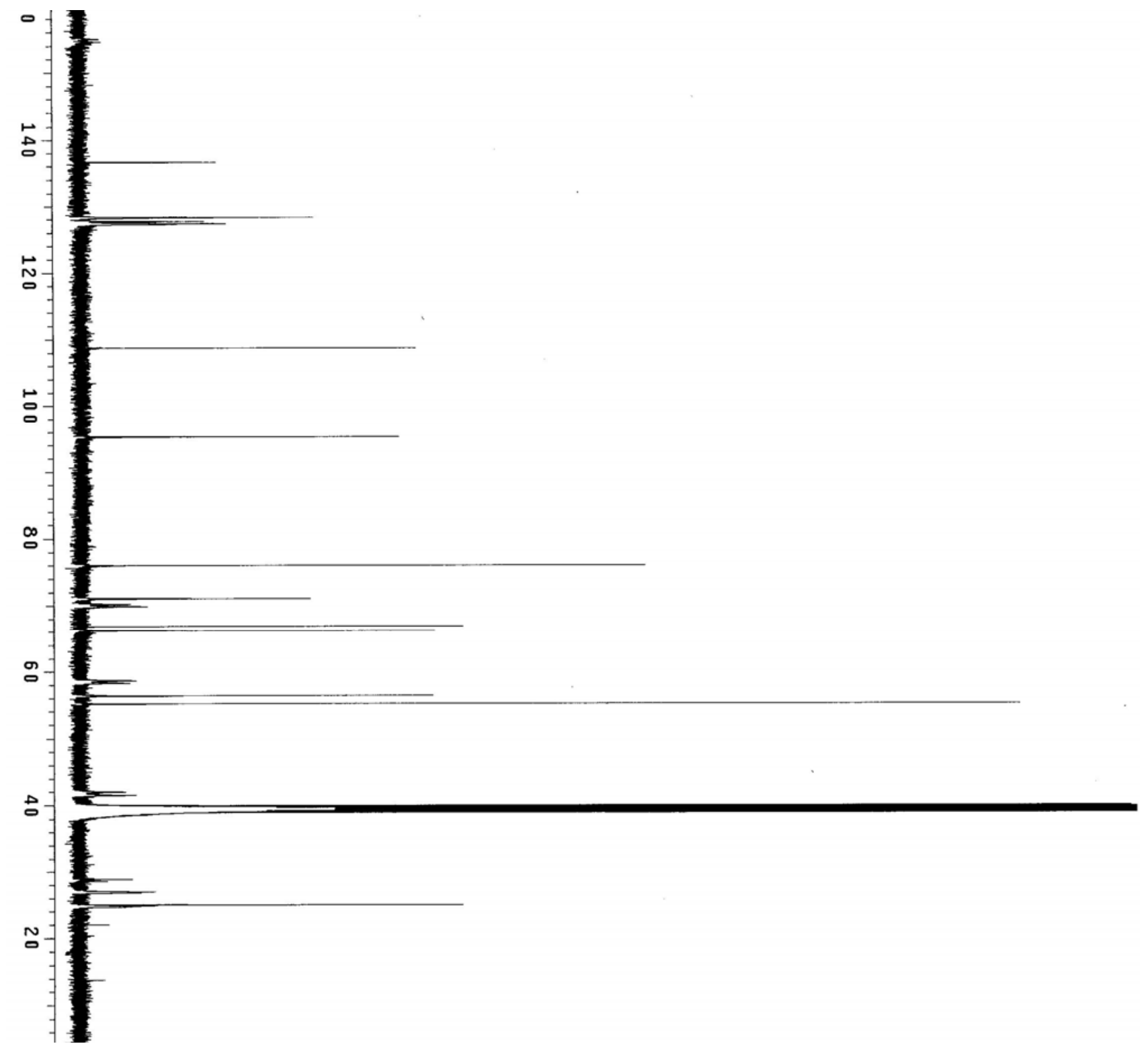



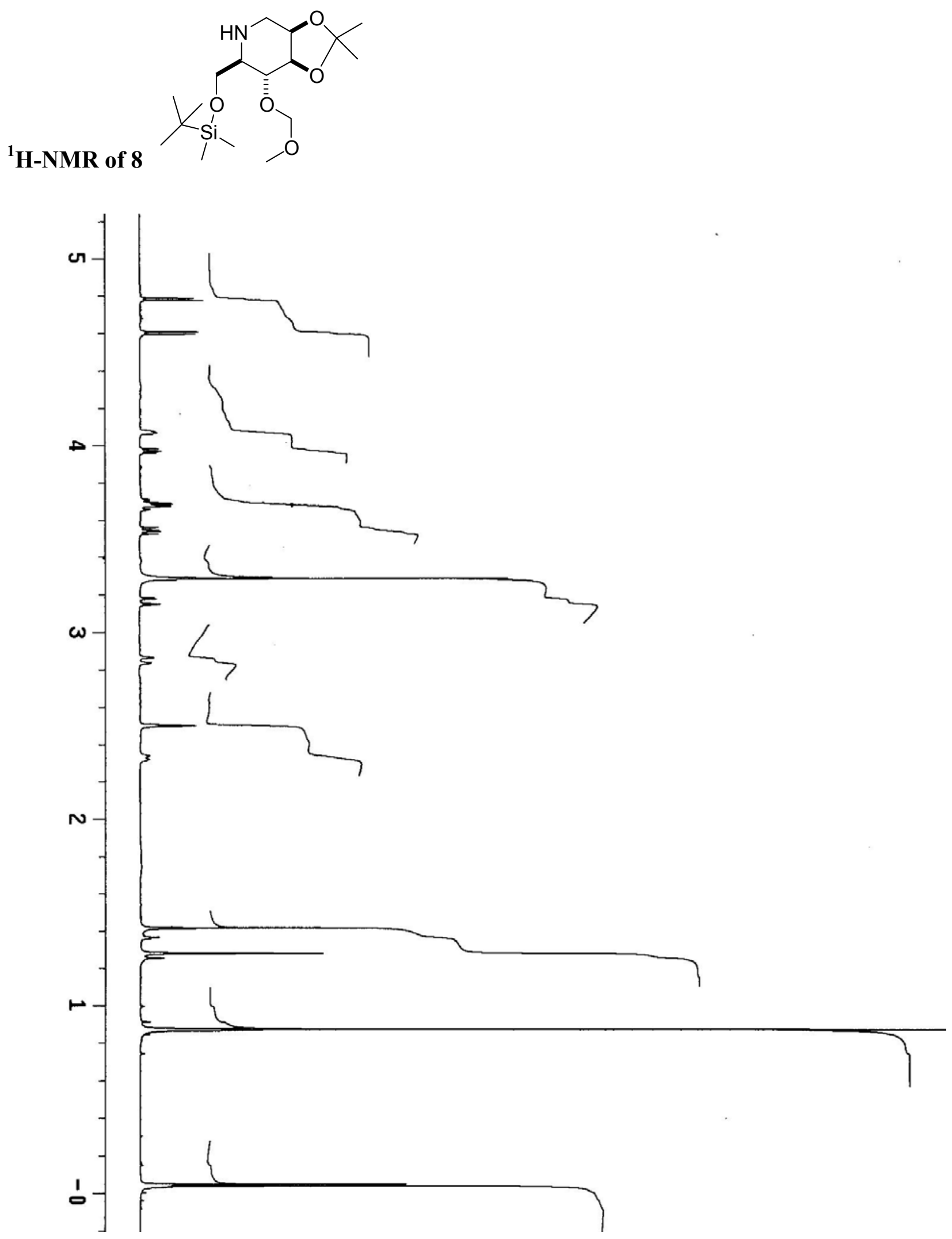

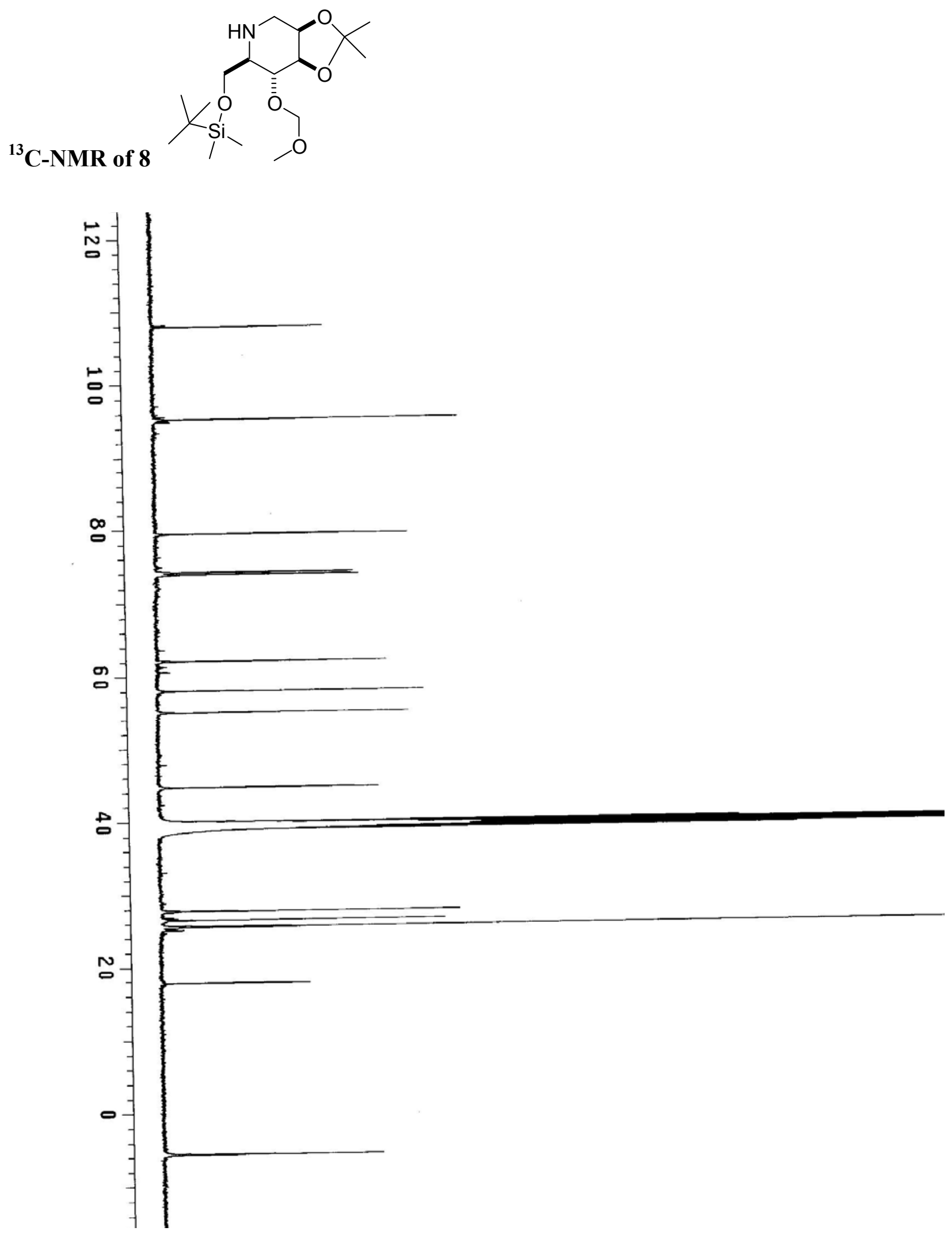

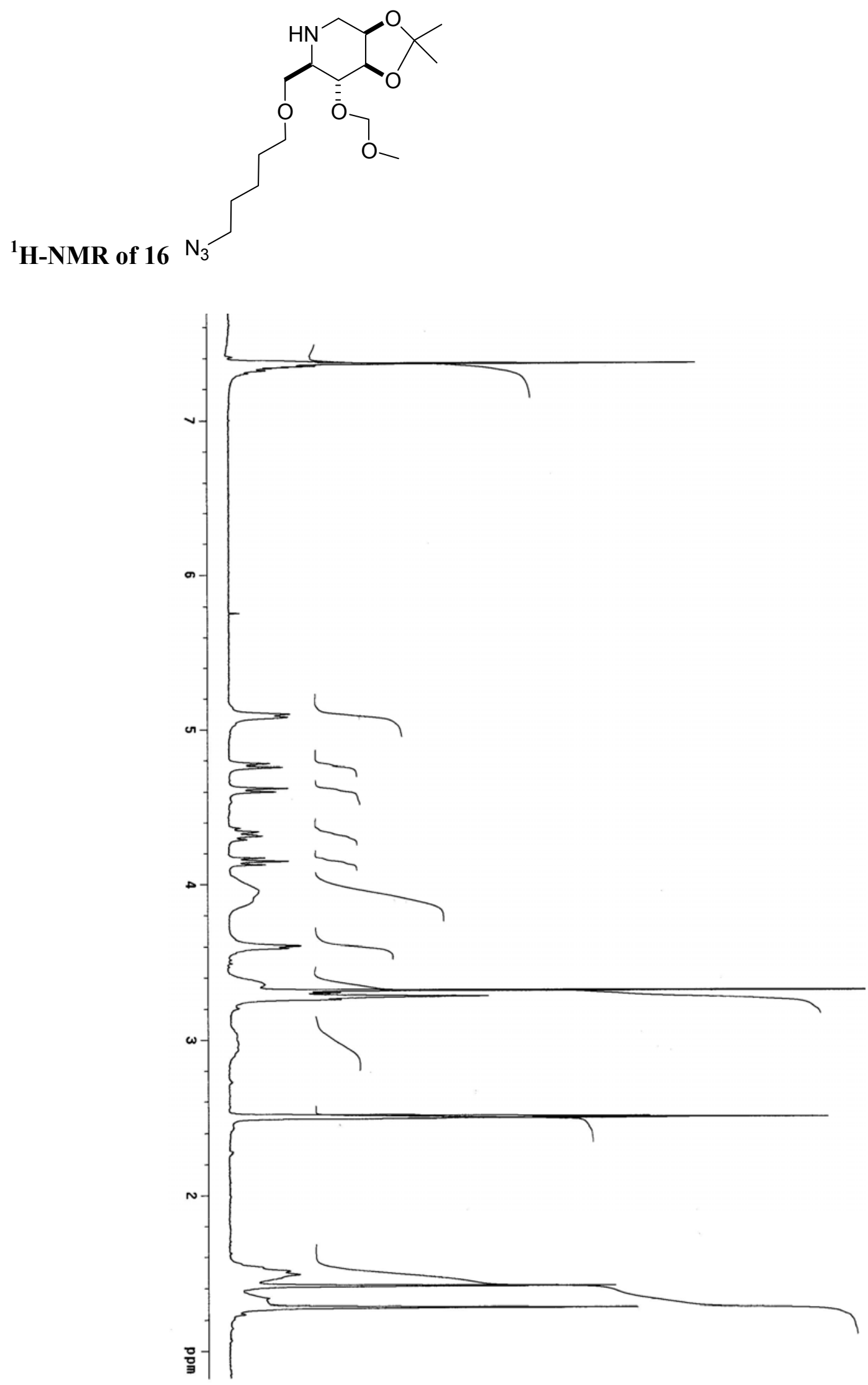

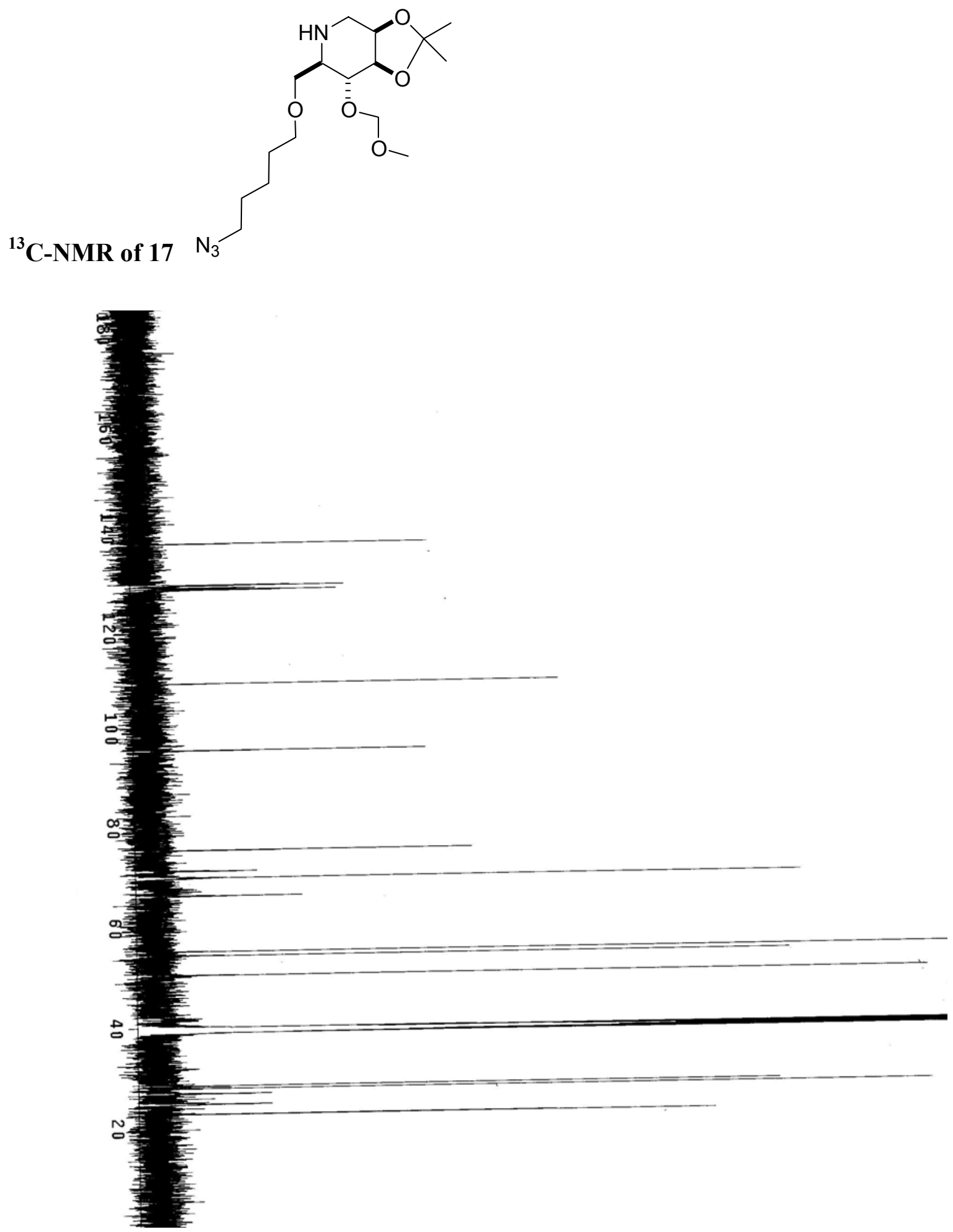


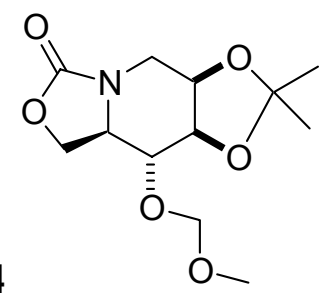

${ }^{1}$ H-NMR of 14

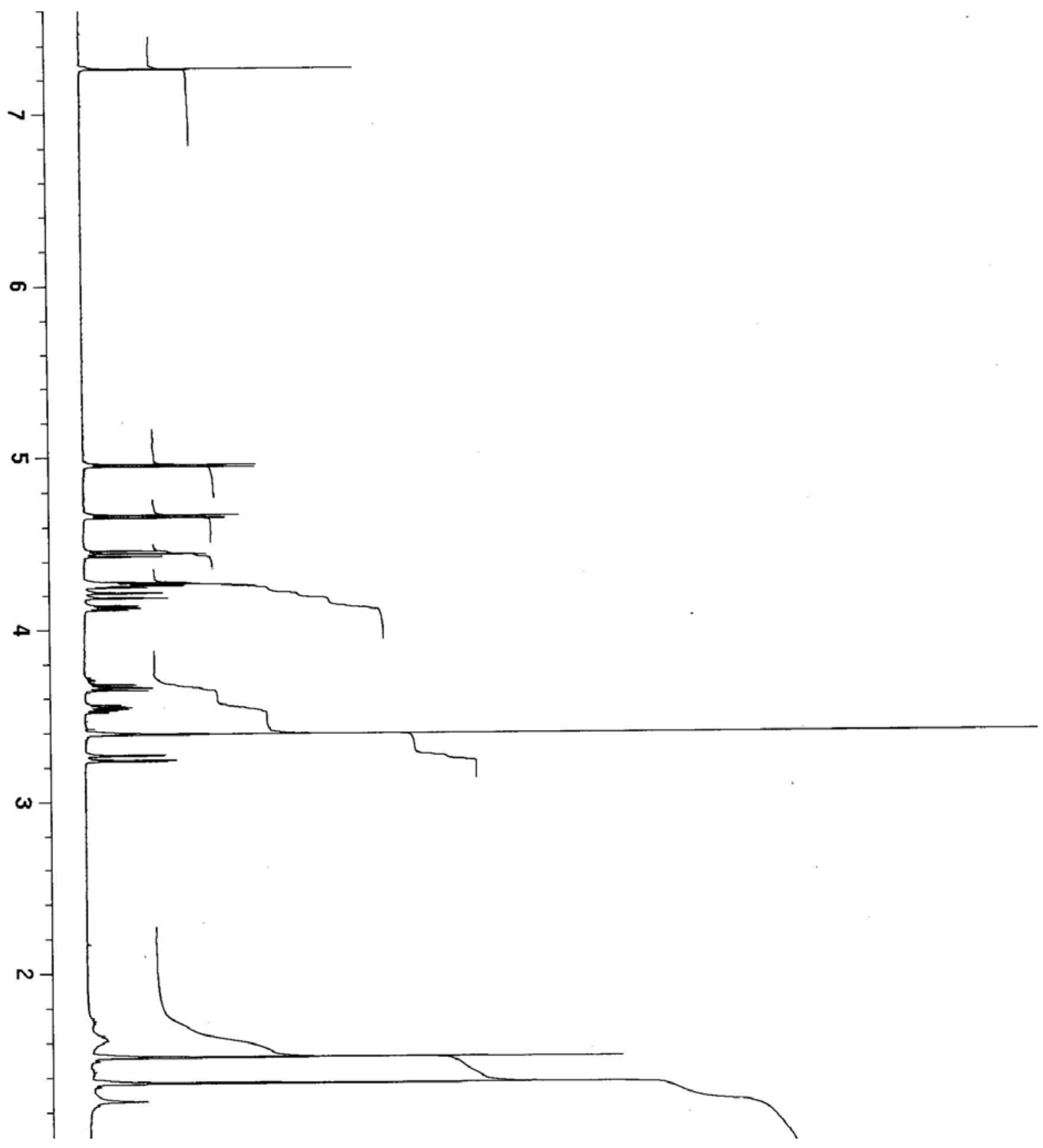


<smiles>COCO[C@@H]1[C@H]2OC(C)(C)O[C@@H]2CN2C(=O)OC[C@H]12</smiles>

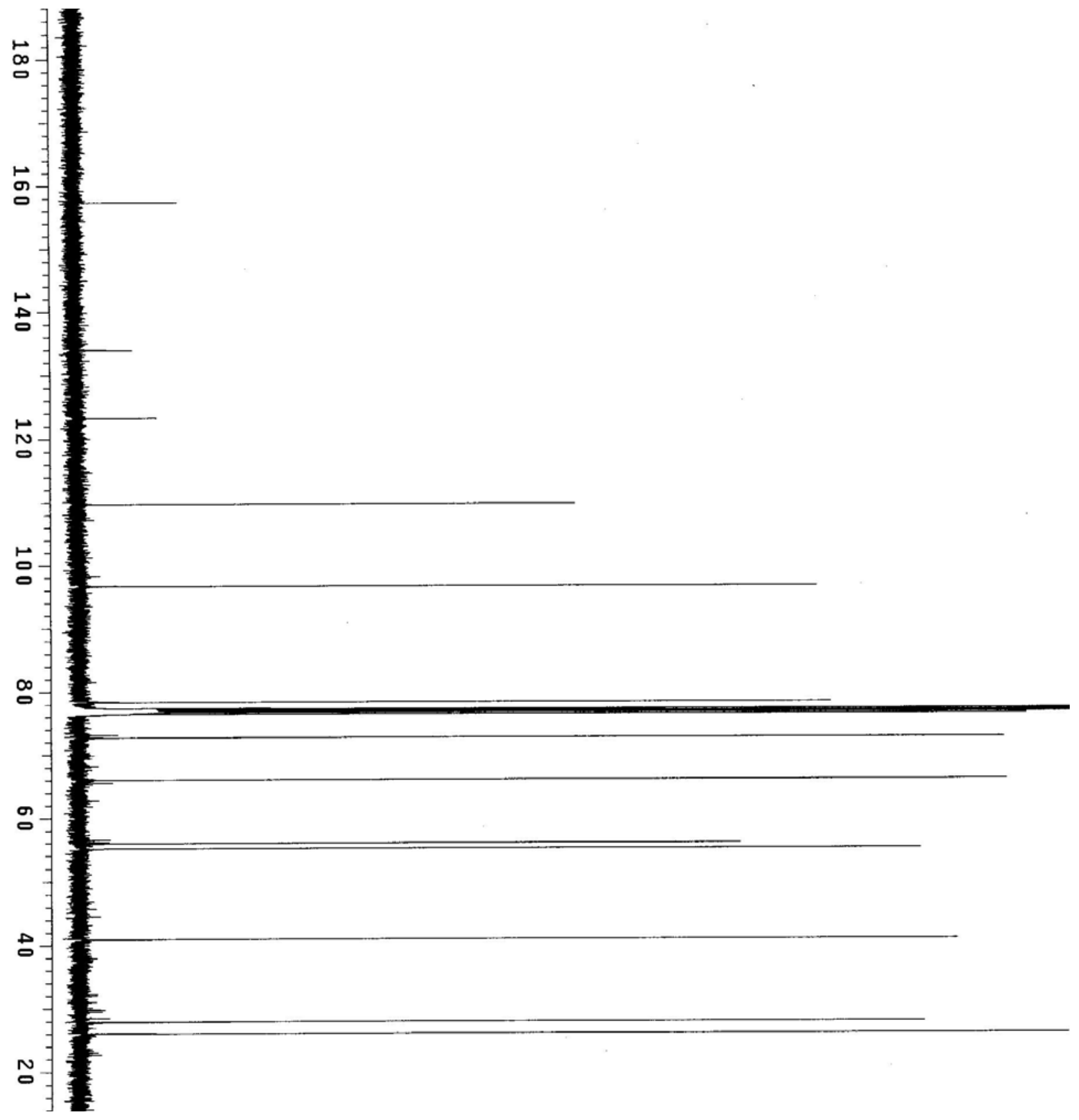



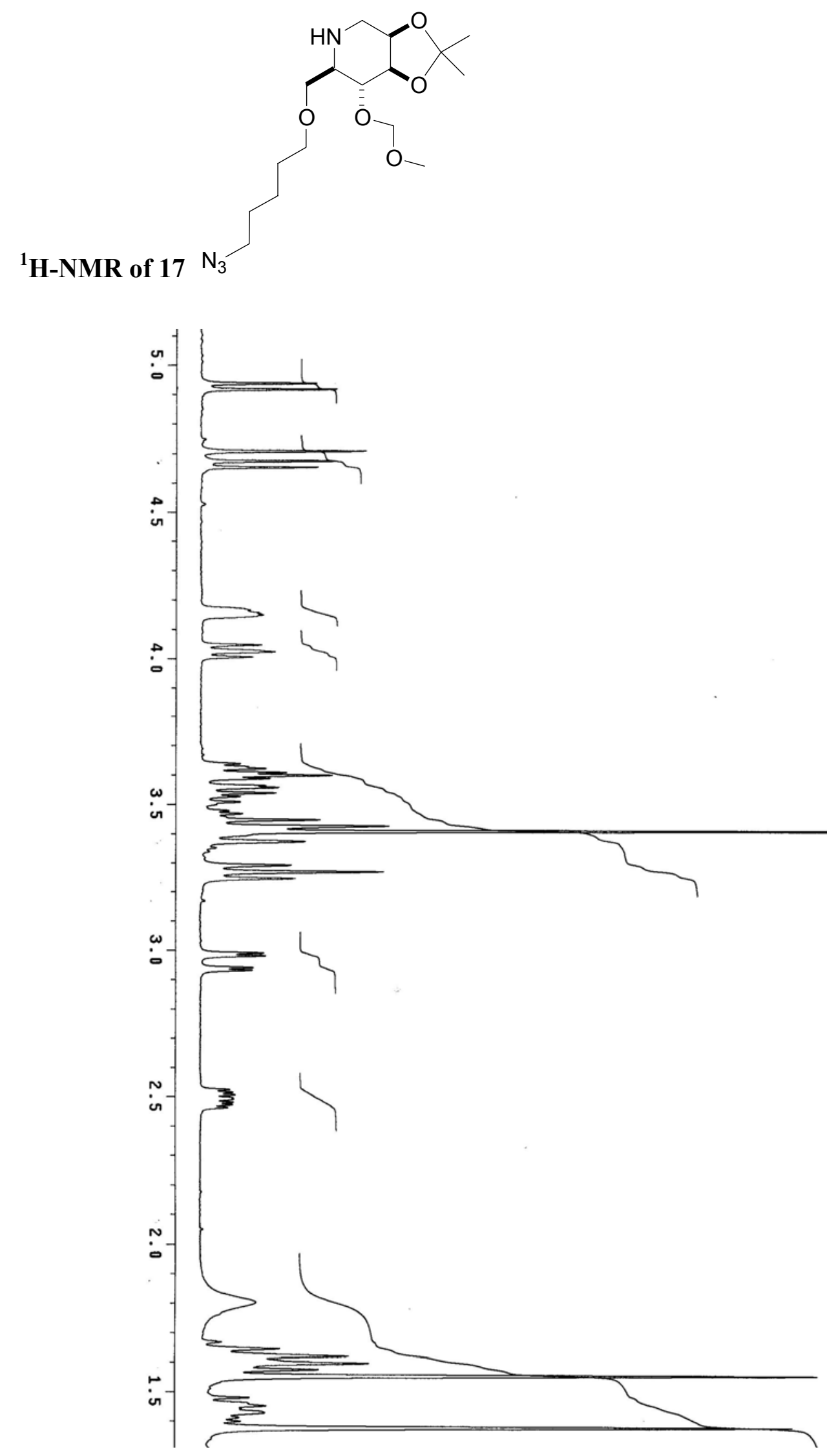

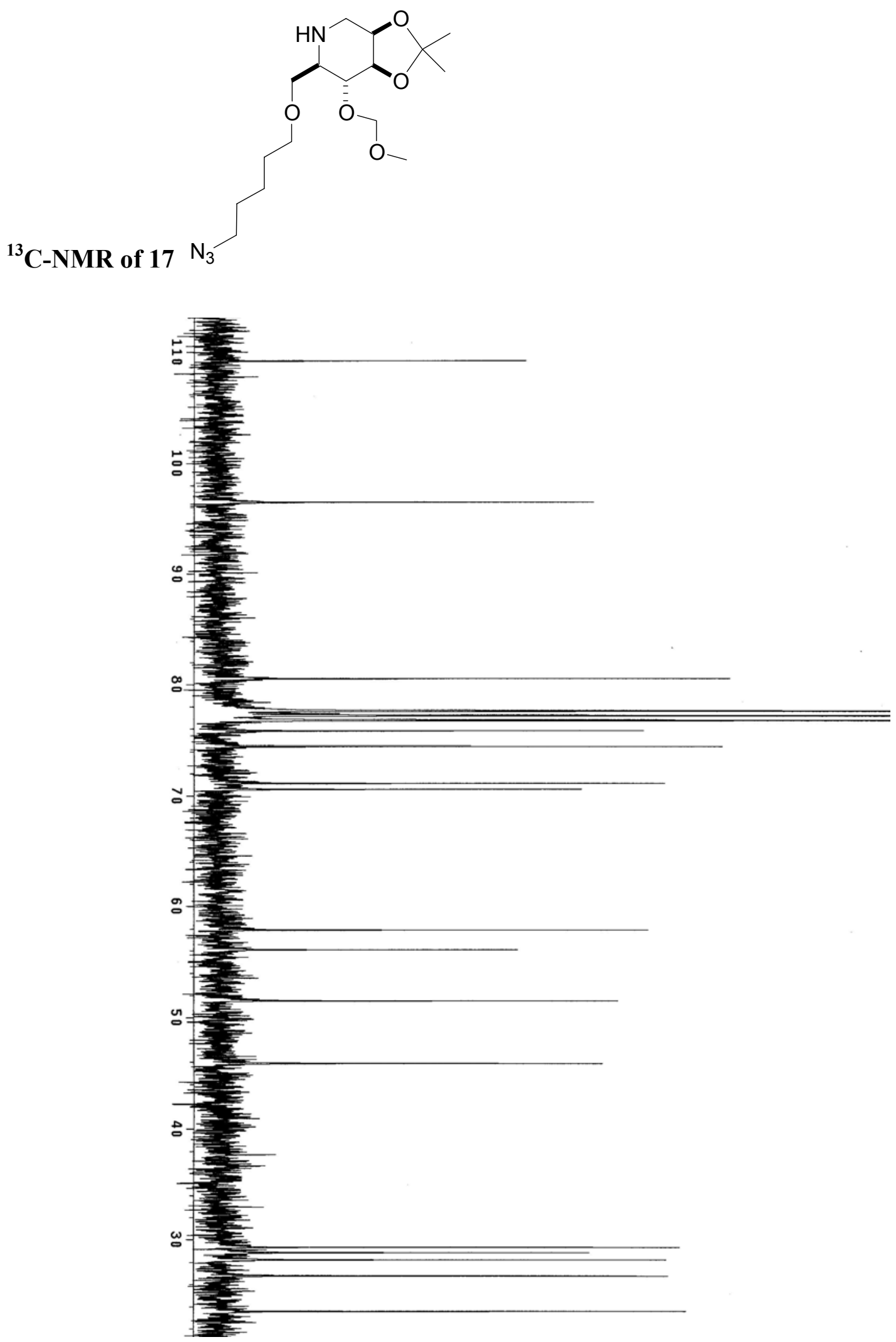
Somatostatin mimetics based 1-deoxymannojirimycin

Gouin and Murphy

S21

${ }^{1}$ H-NMR of 20<smiles>COCO[C@H]1[C@H]2OC(C)(C)O[C@H]2CN(CCc2cn(S(=O)(=O)c3ccccc3)c3ccccc23)[C@H]1COCCCCCN</smiles>

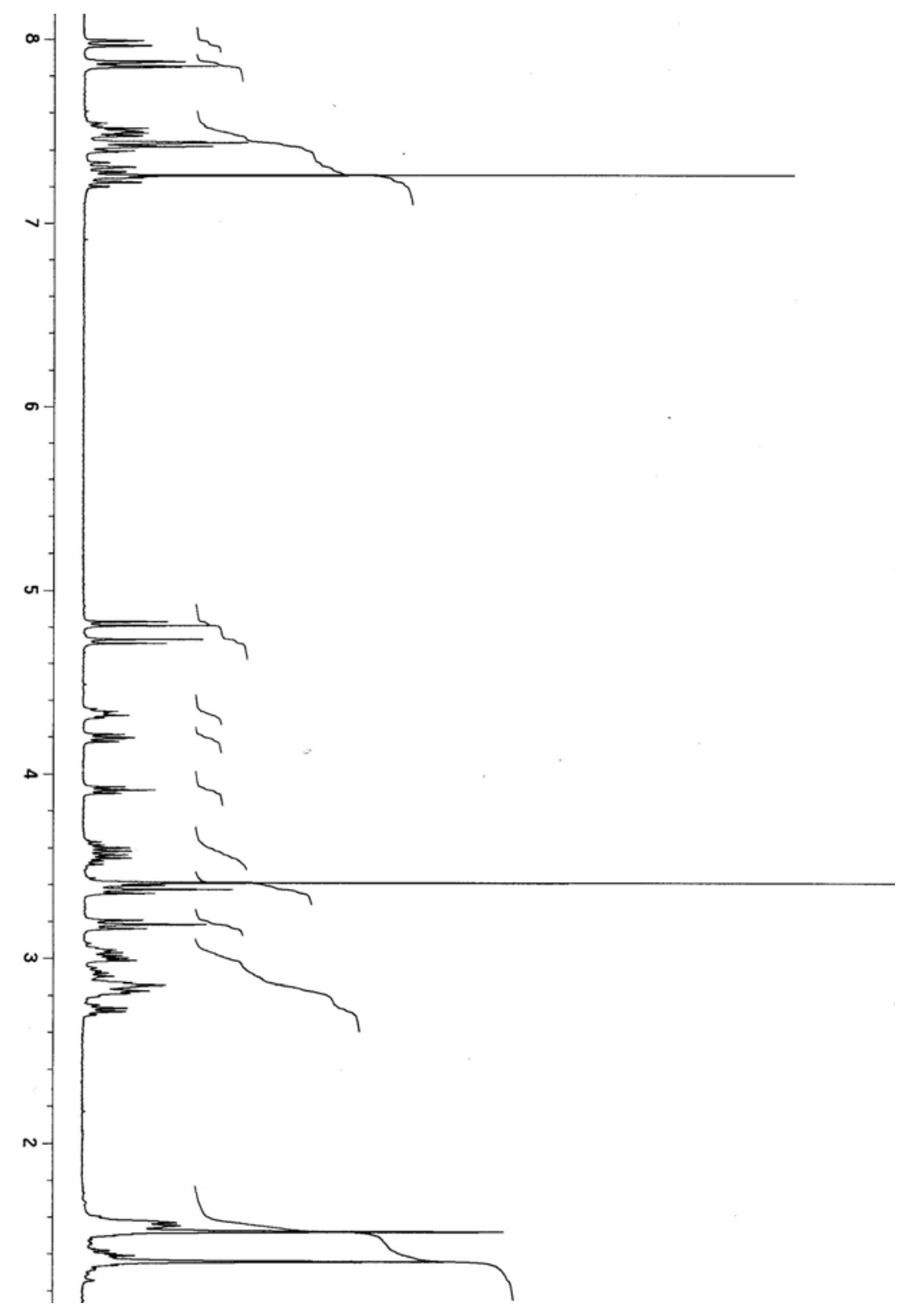



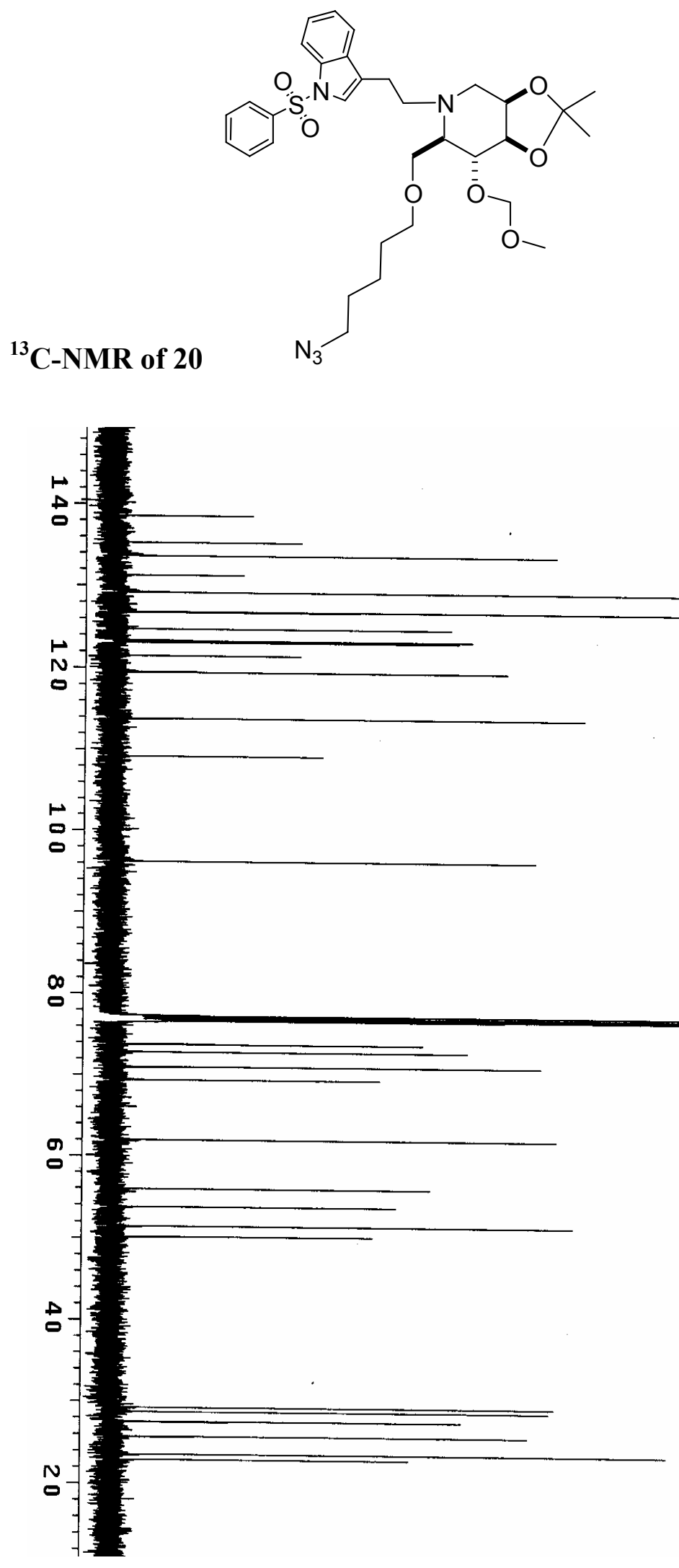

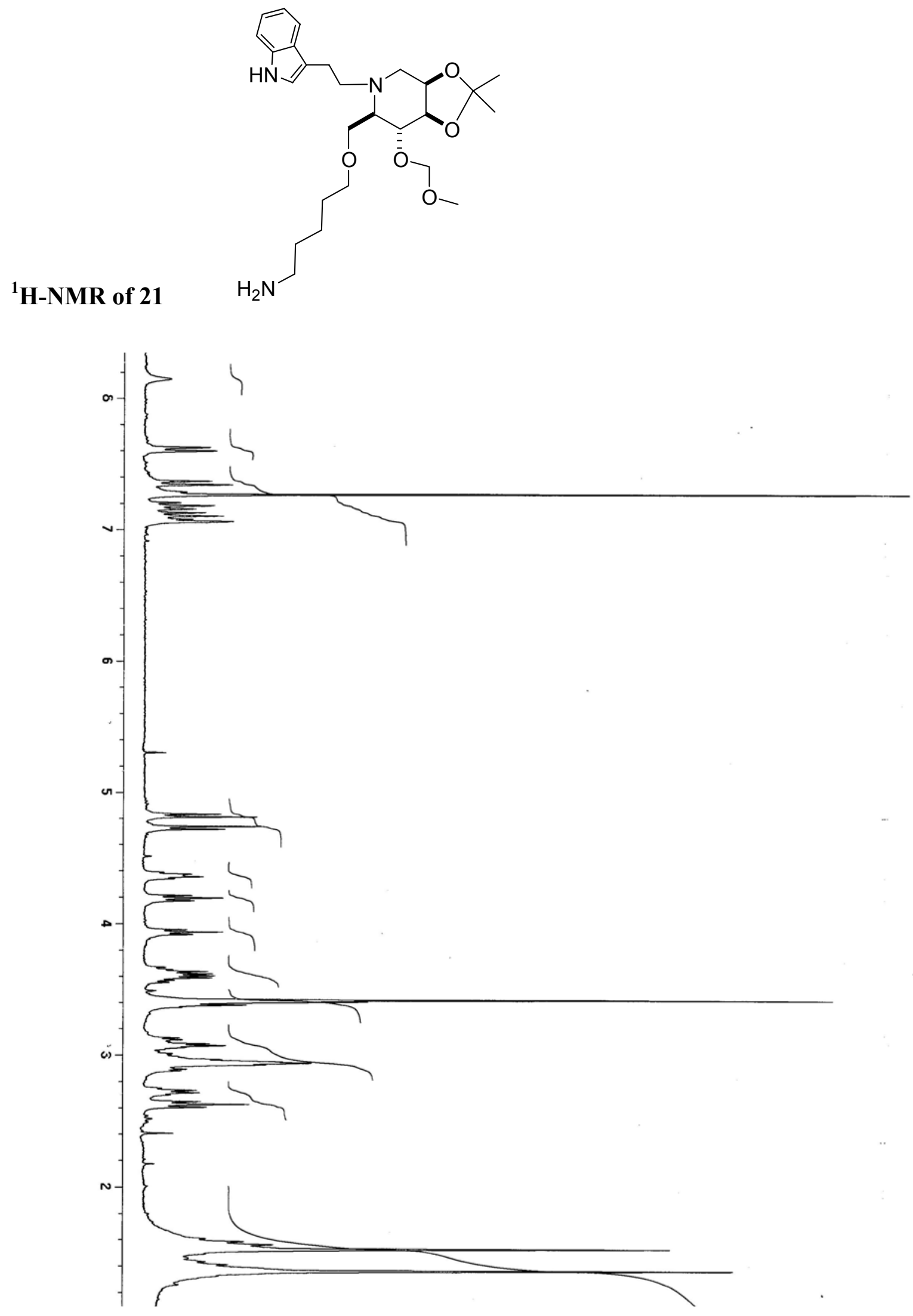

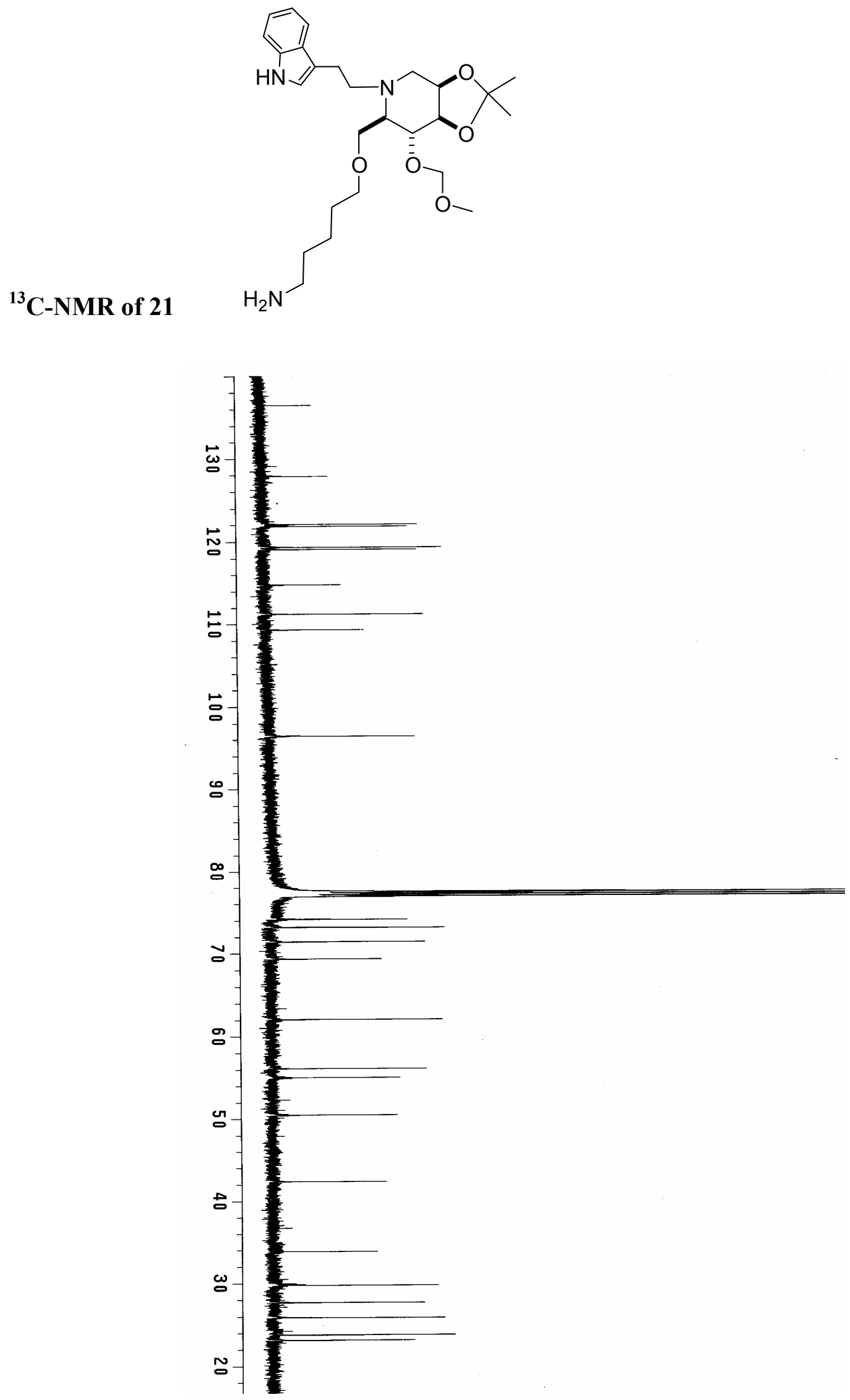

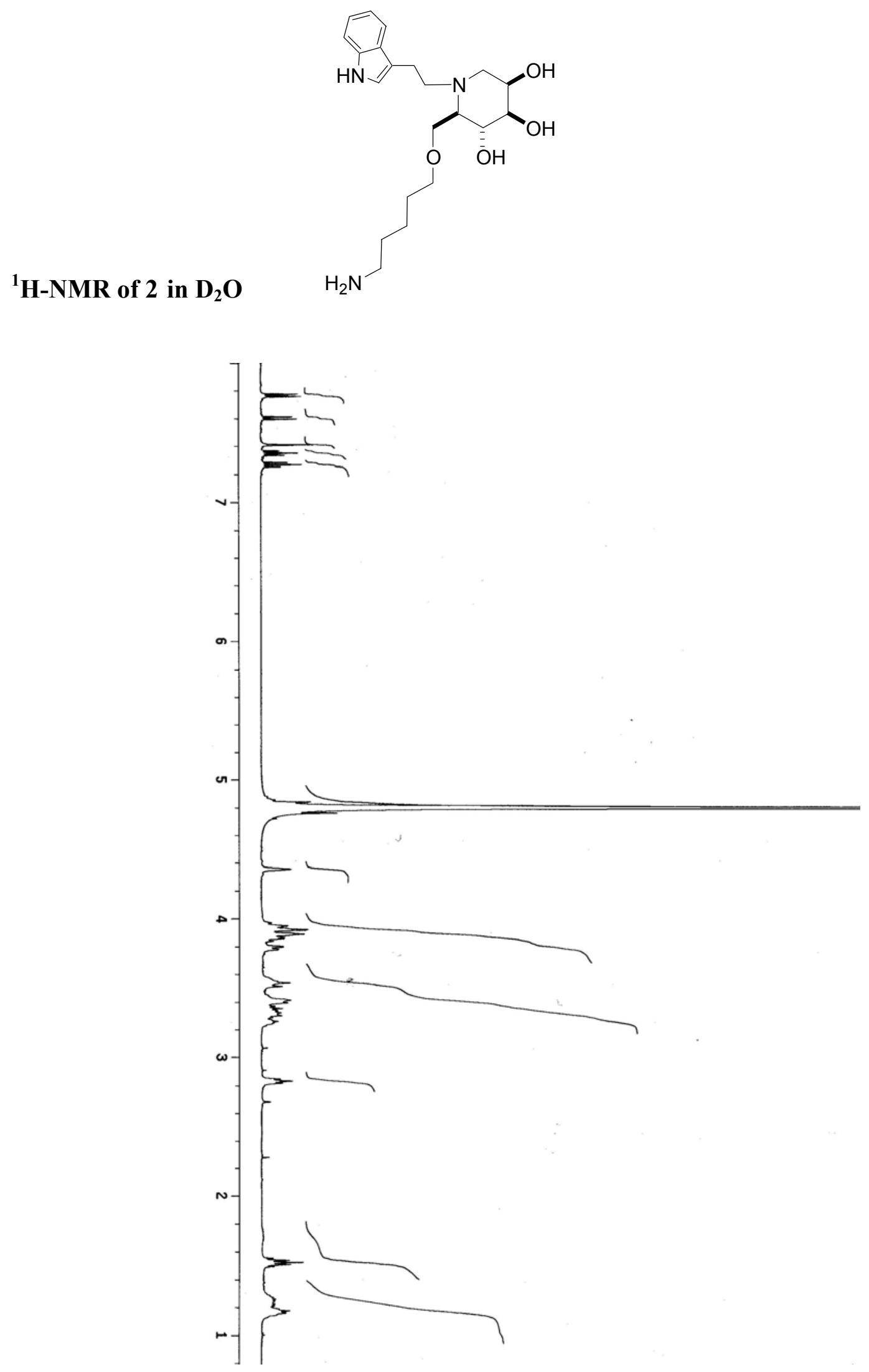


\section{${ }^{13} \mathrm{C}$-NMR of 2 in $\mathrm{D}_{2} \mathrm{O}$}
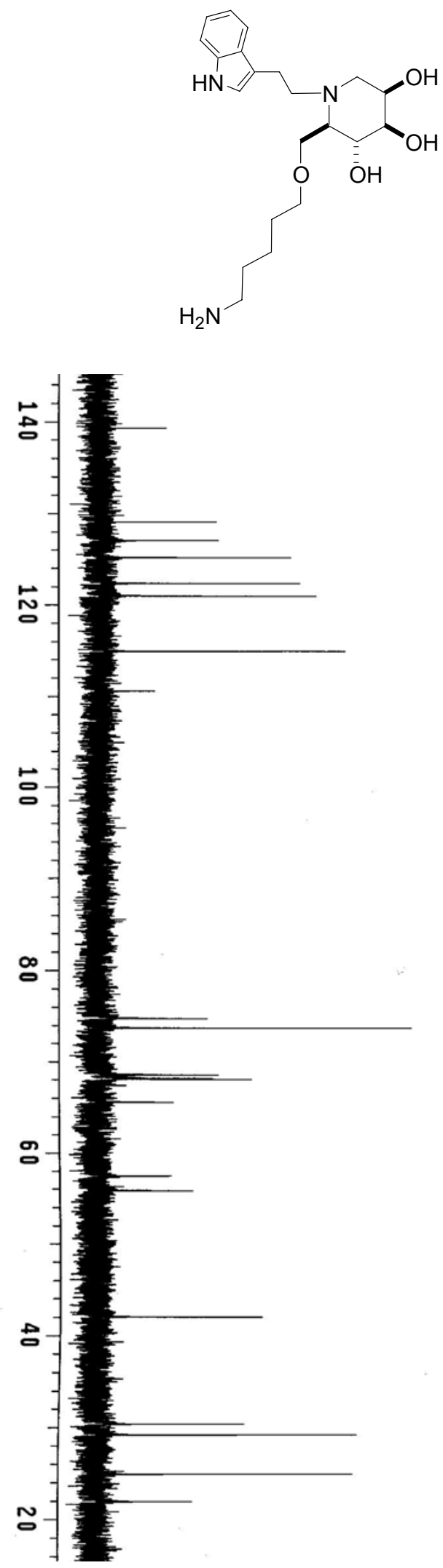